\title{
New hardware engine for new operating systems
}

\author{
Nazjla Ahmadi ${ }^{1, *}$, Mehrad Kaveh $^{2, * *}$ \\ ${ }^{1}$ Department of Computer Science, Islamic Azad University, Ghorveh Branch, Ghorveh, Iran \\ ${ }^{2}$ Department of Computer Science, Islamic Azad University, Mianeh Branch, Mianeh, Iran
} E-mail address: sanandajstudent@gmail.com , alimehrad@yahoo.co.n

\section{ABSTRACT}

Genetic algorithm is a soft computing method that works on set of solution These olutions are called chromosome and the best one is the absolute solution of the $\mathrm{km}$. The m roblem of this algorithm is that after passing through some generations, it may pro d some shromosomes that had been produced in some generations ago that causes reduci the convo seed. From another respective, most of the genetic algorithms are implemente in software and lo works have been done on hardware implementation. Our work implements netic algorithm in hardware that doesn't produce chromosome that have been produced in previc generations In this work, most of genetic operators are implemented without producing iterati hromos mes and genetic diversity is preserved. Genetic diversity causes that not on don't this converge to local optimum but also reaching to global optimum. Without any on roposed approach is so faster than software implementations. Evaluation results also show the ror ssu proach is faster than hardware ones.

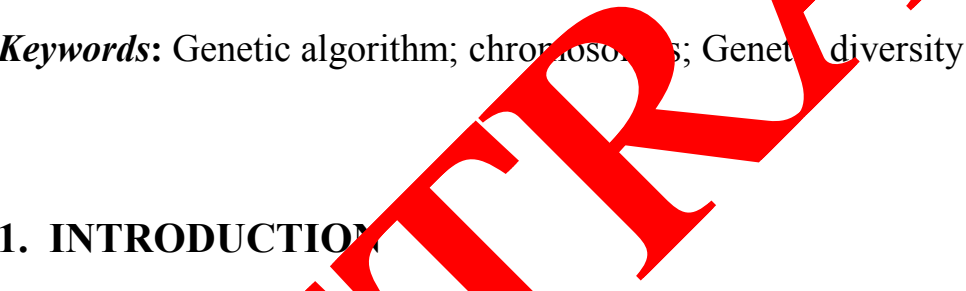

Genetic alsonthm is a s omputing method that works on set of solutions. Each of these solution are c led chrop osome and each population consists of a certain number of them. By $a_{1}$ ir $g$ som operators like selection, crossover, and mutation on the chromosames or rent $\mathrm{p}$, pulation, the next generation is produced.

In GA a a a ned above, it is observed that by passing through a number of ge. tion some chromosomes may be produced that are the same as the chromosomes in the pr us gomations. It is clear that these chromosomes are not suitable ones because they were eln ted in the previous generations because of low fitness value. The main problem of these ch omosomes is increasing calculations of each generation because GA operators in current generation are applied on chromosomes that were produced and deleted in the previous generations [1].

This process also causes decreasing of convergence speed toward problem solutions. Assume two individuals in generation $\mathrm{N}$; produce two offspring by applying crossover and mutation. Now in generation $\mathrm{N}+1$, if these two offspring recombine together, it may be produced chromosomes that are similar to ones in generation N, consider the Figure 1 [1]. 


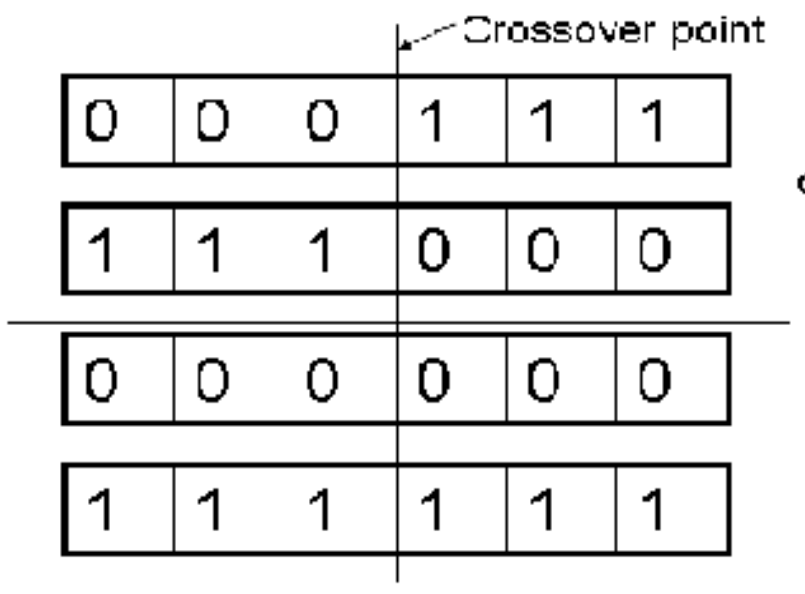

Chromssomes of generation $\mathrm{N}$
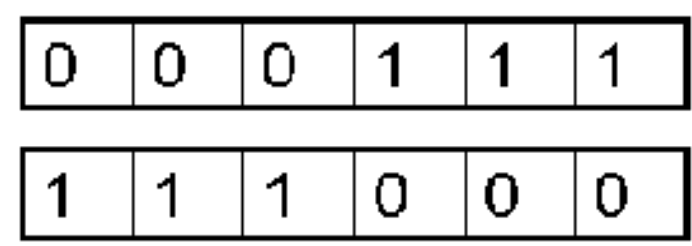

Figure 1. Producing of repeated thromosomes [1].

In [1] individuals are divided into to groups

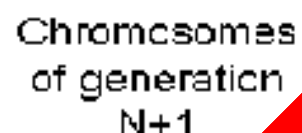

$\mathrm{N}+1$

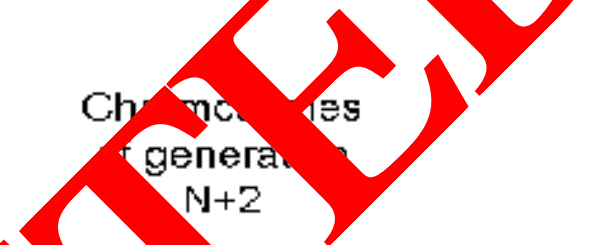




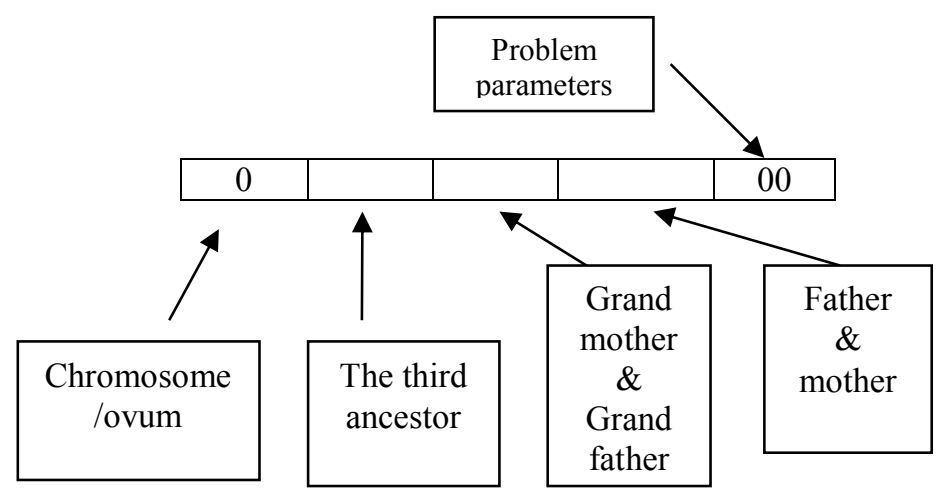

Figure 2. The structure of individuals.

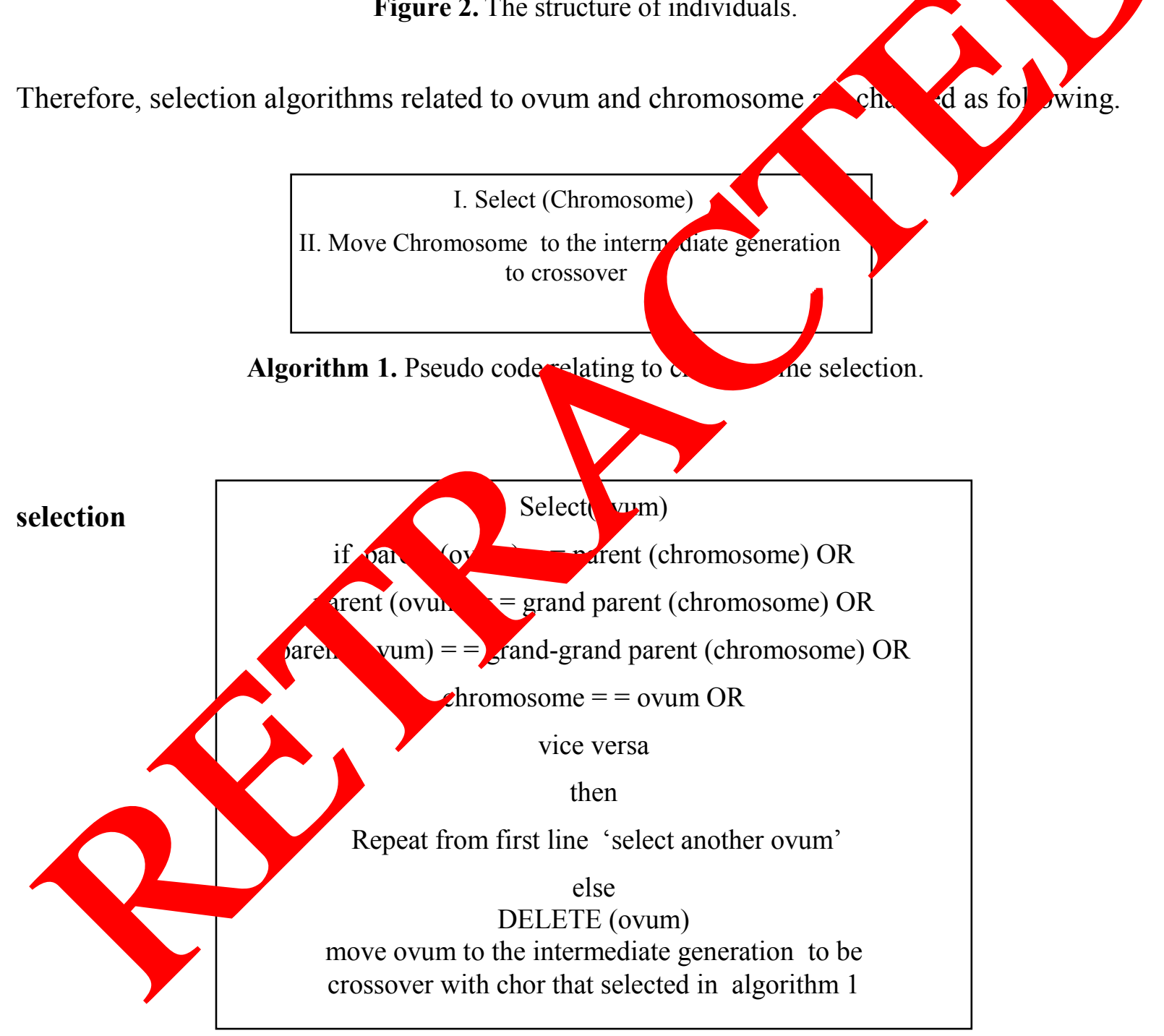

Algorithm 2. Pseudo code relating to ovum.

Also in [1], the recombination method put the information of recombined individuals into next stage of produced individuals. Figure 3 shows the process of crossover method. 


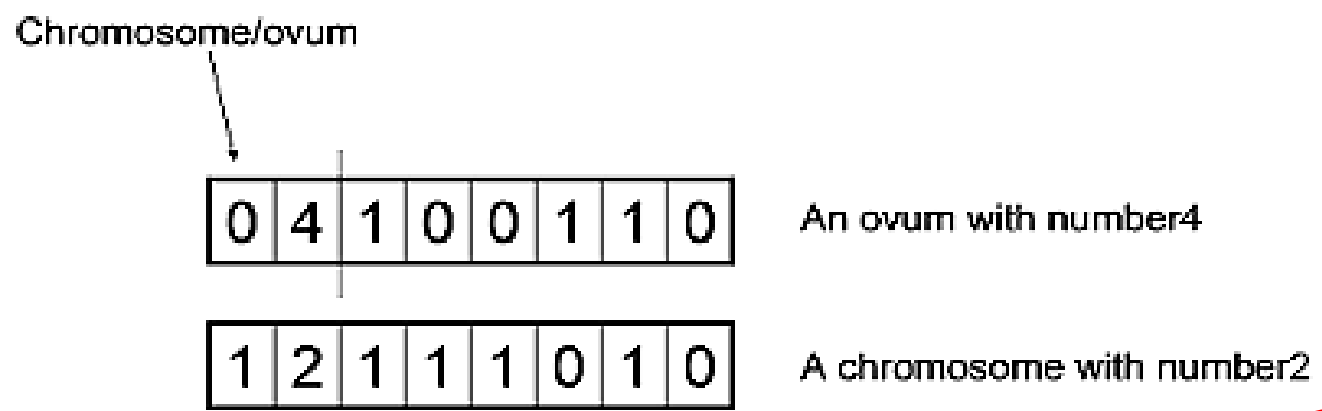

For another respective, almost all of the gen gorithms are implemented in software. But in this work, we propose para genetic algorithm and compare it with pre iou sove The evaluation results show the usefulness of the researchers enternmice work. Previous works $[1,2,4,5]$ can be divided into three categories. 1- Those that mpl ent fitn sy function on hardware and other parts of genetic algorithm like crossor vuta on and selection in software. The disadvantage of this method is that hardware d puds a provum. 2- Those that implement fitness on software and other operators in $b$ ware. In method hardware is independent of a problem but the running time is inc ase 6,7]. 3- nose that implement both fitness and operators in hardware $[2,4,167$ his w w proposed an approach that improves this type of method for new appro\% th to standard netic algorithm [1]. This paper divided into 5 sections. In section 2, $t^{\prime}$ prop and aproch is described and in section 3, architectures of genetic operators are ained. ections 4, 5 evaluate the results and draw some conclusion, respec

\section{OV ALL MEW OF PROPOSED ARCHITECTURE}

The ofference of genetic algorithm with other soft-computing methods is individuals' representation. Individuals in genetic algorithm are a stream of binary bits that are very attractive to hardware implementation. The selection, crossover, and mutation are generic operators and never depend on the problem types. These characteristic make them easy to implement. The main problem of implementing of genetic algorithm is fitness function. For this reason, to calculate it, hardware of neural networks is used $[2,9,10]$. Ideal estimation of each individual can be obtained using this network. Another reason to use neural network to compute fitness is its simplicity and stochastic representation of signals that reduces the 
hardware area. In figure4 the overall view of proposed approach is showed. Genetic operators of this architecture are as follow.

- Selection operator: Rolette wheel \& tournament

- Crossover operator: single point \& two-point

- Mutation operator: bit complement

- Replacement operator: steady state \& generalization

The main and novel contribution of this work consists of using all genetic operators in hardware implementation without producing repeated individuals in alternative pricm ss. Hardware implementations of these operators are described in section 3 . The $p$ in advant. of this architecture over previous works [16] is using fitness of next generation splaceme operator to avoid re-computing of fitness function in each generation. Fo ctirs rea fitn os of each chromosome is kept in bank of registers and individuals' fitne sof ne gene on is provided as input. It is necessary to remind that each register of regist ar. invo reall part of individual structure mentioned above. When replacement op ator is ady st $\mathrm{e}$ and the fitness of input chromosomes are greater than ones that kep the regist $1 \mathrm{k}$, the input chromosomes are replaced. It should be noted that Fitnes of ea chromos ome in the first population is considered as 0 [1].

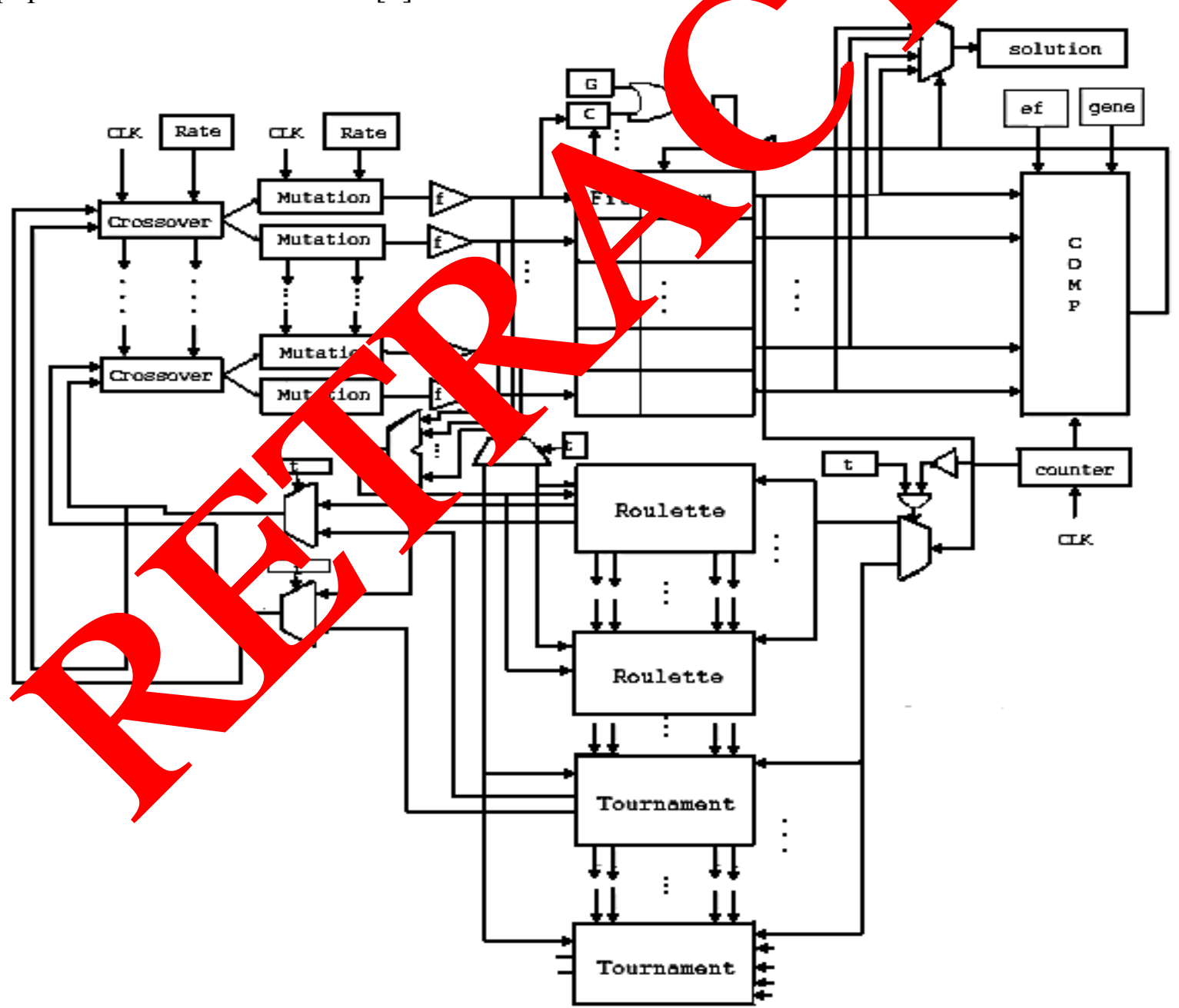

Figure 4. Overall view of architecture [2]. 


\section{OPERATORS ARCHITECTURE}

The architectures of selection, crossover, and mutation operators and shard memory are described in this section.

\section{A. shared memory for generational population}

All of the chromosomes are kept in synchronized bank of registers. Each register keeps individuals as described in Figure 2. These registers can be updated and written. The registers can be writte by the following steps.

1- Whenever the comparator output is 0 and replacement operator is generality

2- Whenever the comparator output is 0 and replacement operator is fitness measurements of chromosome in next generation are greater than generation.

It is necessary bearing in mind that the output of comparato is 0 it he num or of generation (gene) is greater than counter or fitness of all chromose ne $\mathrm{cos}$ than ithess. In such condition genetic algorithm continues to work and current eneration repla ed by next generation.

\section{B. Random number generator}

This generator is used to produce crossover \& feedback shift register are used. More details about $[11,12]$.

\section{Roulette selection components}

In this subsection the roulette opera or to component are generational chromosomes $\left(\mathrm{I}_{1}, \mathrm{I}_{2}, \ldots, \mathrm{I}_{\mathrm{n}}\right)$, fitne uncti $\mathrm{n}\left(\mathrm{F}_{1}, \mathrm{~F}_{2}, \ldots, \mathrm{F}_{\mathrm{n}}\right)$, and cumulative sum of these fitness. This component so dividuals and passes it to crossover unit. The hardware of this component is sy to hplemen for more details see $[1,16]$.

\section{Tournament selection}

One of the nove tributions this work is tournament operator. This component takes several chrom ome gether with their fitness and their information and output the fittest one that no sutisfy any hefollowing conditions.

- $\operatorname{parent}(\mathrm{g}, \mathrm{m})==$ arent (chor)

- parent(ovun = grar a parent (chor)

- arent vum) $=$ srand-grand parent (chor)

- o previous generations of chor

- chor the previous generations of ovum

- $\operatorname{parent}($ chor $)==$ parent (ovum)

- $\operatorname{parent}($ chor $)==$ grand parent (ovum)

- $\operatorname{parent}($ chor $)==$ grand-grand parent (ovum)

- $\quad$ chor $=$ ovum

Algorithm 3 shows the hardware algorithm of this unit. 
1. Choose 3 individual in the population

2. Compare them according to their fitness's

3. Output the best one to individual1

4. repeat step 1 to 3 to select the second one

5. If two selected individuals doesn't satisfy any of the conditions, put them into final individuall and final individual 2

Algorithm 3. Algorithm of tournament selection.

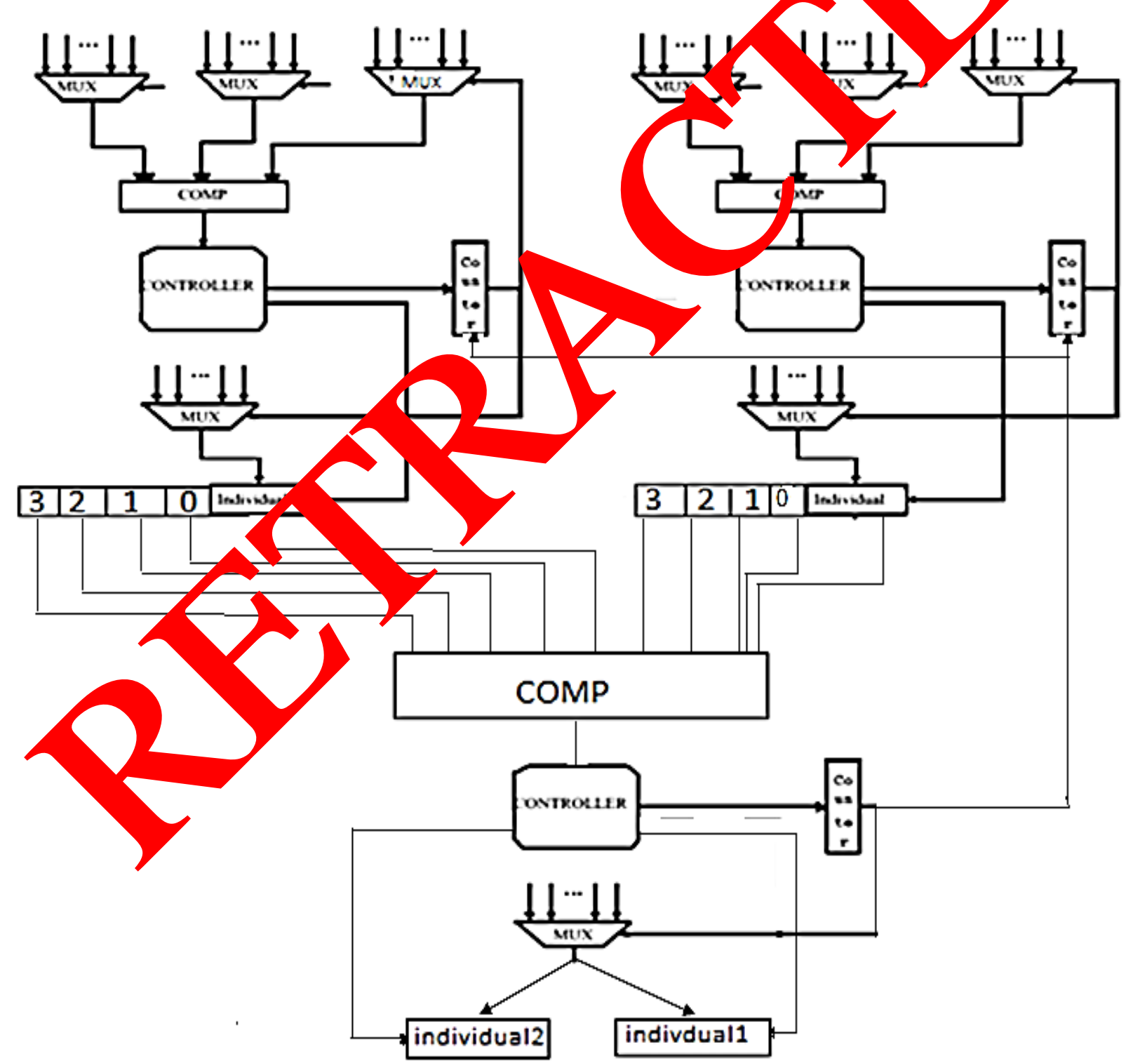

Figure 5. Architecture of tournament selection. 
In this algorithm, three chromosomes are selected and raced to be one of the individual intermediate generations. This routine is also repeated to select the second chromosome. The hardware implementation of this component is shown in Figure 5. Finally, if the selected individuals don't have any above mentioned properties, they are selected and put into final individual1 and final individual 2.

The synchronized bank of registers that keep the individuals are input of the multiplexers, comp unit compare the fitness of these chromosomes and also relationships between them. After passing from these steps, comp unit outputs the number of those individuals. This number is applied to selection signal of multiplexer in the middlo of the Figure 5. In other word, the appropriate chromosome is selected and sent to indi caualn, same process is repeated for selecting ovum. After that, the properties of two dividuals a compared to judge about their relationship. In this research iterative process is lement using state machine (controller) [1,16].

\section{E. Crossover component}

Another contribution of this work is implementing a unit or new cover asing both one point and also two-point recombination. To crossover tly, the com Number Generator produce a random number say $p$. if $p<\mu_{\text {rate }}$ the cross operator is applied. In the case that crossover applied, the bits of the less sign half on randomized number is used as the first crossover point and the most signif cant part as the sf cond one. It should be reminded that in the single-point crossover, the bits the most sifnificant part are considered as 0 . Finally, the information of selected individu are shifte to next stage of produced individuals like as Figure 2. Figure 6 shows the hardwa nnl nentation of crossover.

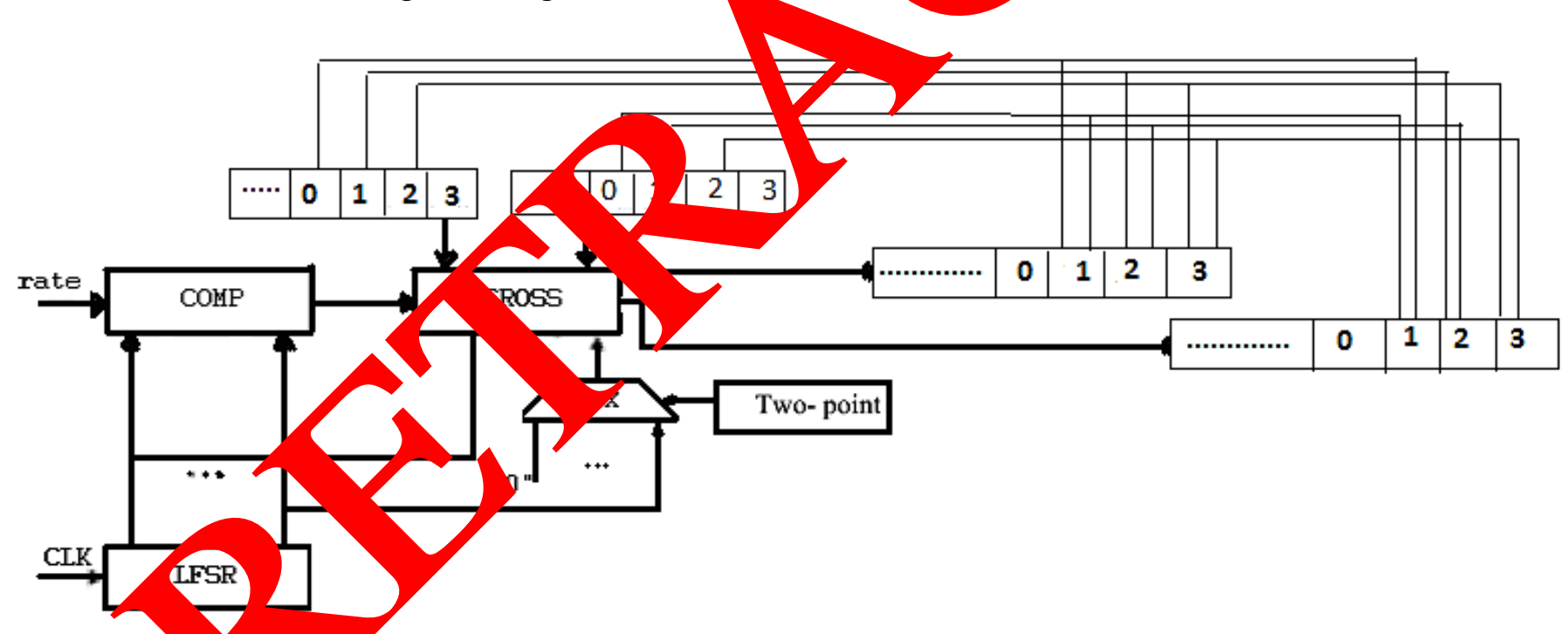

Figure 6. Hardware of crossover operator.

In this hardware if the value of two-point register be one, two-point crossover is applied; otherwise, one-point crossover is applied.

\section{F. mutation components}

This component has architecture like as crossover. To implement this component, a random number is produced using Random Number Generator. When this value is less than $\mu_{\text {rate }}$, the mutation operator is applied. For more detail see $[1,16]$. 


\section{G. Fitness components}

For the reason that the fitness function will not be depending on a problem, the neural network is used to estimate fitness measure [10] in this work. In [10] neural network has been implemented using stochastic signals and therefore reduces very significantly the hardware area required for the network. For the genetic hardware implementation, the number of input neurons is the same as the size of individuals in population. The output neuron is augmented with a shift register to store the final result. The training phase is supposed to be performed before the first use within the hardware genetic algorithm.

\section{EVALUATION RESULTS}

Hardware for new genetic algorithm was simulated and ther programmo int a Sparatan3 Xilinx FPGA [13]. To assess and evaluate of proposed ar ectur func son in [14] was used to maximize. This function was also used in previ th to eva ate their hardware implementation for genetic algorithm. This function $\mathrm{j}$ 's follows.

$$
\mathrm{F}(\mathrm{x}, \mathrm{y})=21.5+\mathrm{x} \sin (4 \Pi \mathrm{x})+\mathrm{y} \sin (2 \Pi
$$

$$
-3.9 \leq x \leq 12.1
$$

$$
4.1 \leq y \leq 5.8
$$
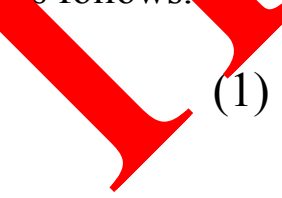

It is clear that this function is not easy to max ze and is a very attractive metric to evaluate and assess of proposed approach. 1 raluationresults are shown in Table 1.

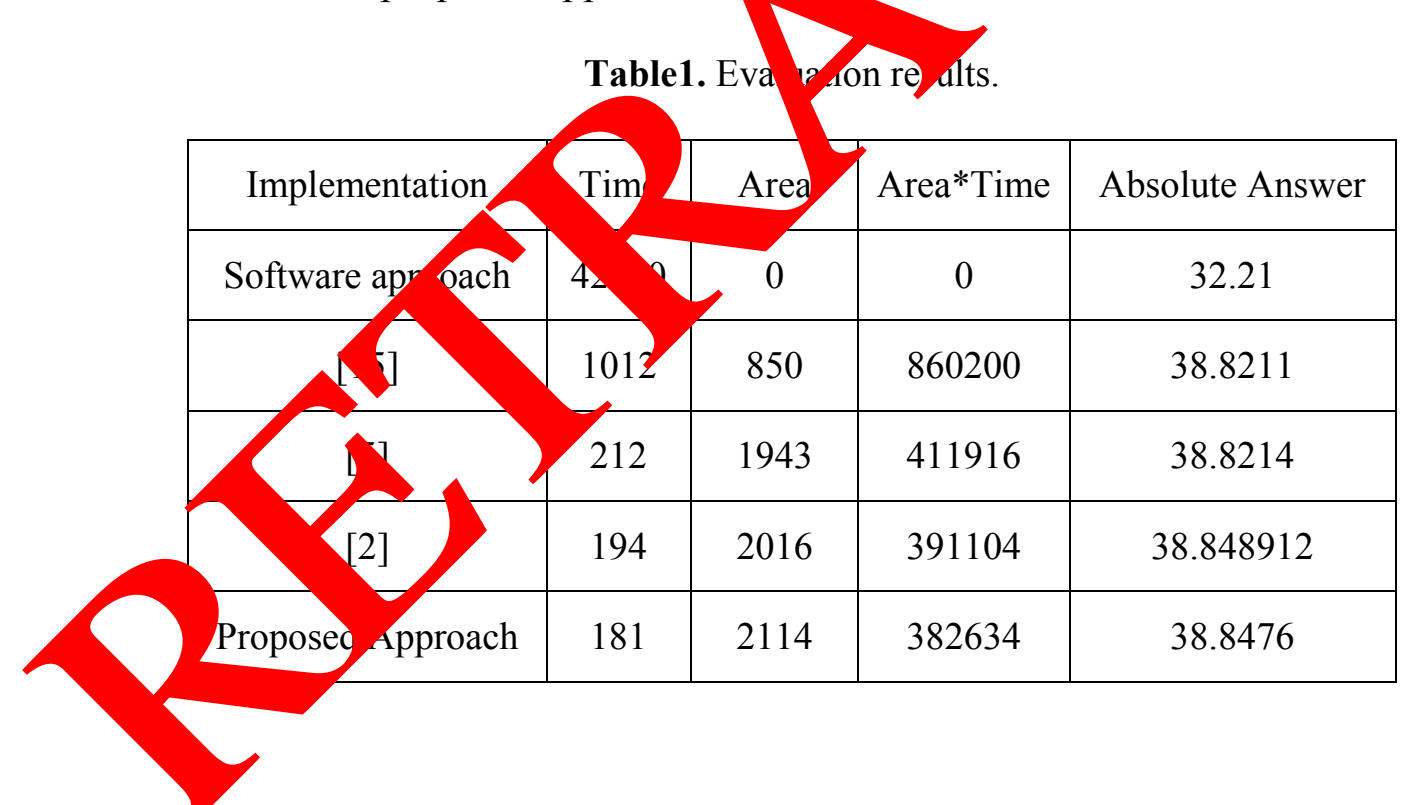

In this table the area is expressed in terms of CLBs and the time is in second, also previous works including the software and hardware implementation and our proposed approach have been compared.

The advantage of hardware approach to software approach is speed up. Therefore, in this work, besides software implementation, hardware implementations are studied and evaluated. It is clear that the area required by this work is more than $[5,15,16]$. From another respective, our proposed hardware is faster than $[1,15]$ and also approximately is faster than [16]. The main advantage of proposed approach over $[15,16]$ is applying almost all of the 
genetic operators that result in genetic diversity. In the [16] only roulette selection, two-point crossover and mutation have been implemented, but in our work and [1] not only have been these operators implemented but also tournament, one-point crossover, and steady state replacement have been designed and implemented. In our work, unlike $[1,5,15,16]$ producing of the iterative chromosome is avoided that causes this approach to increase in convergence speed in comparison to other approaches $[1,5,15,16]$. In our work like [1] the operators can be changed in the next generation by changing a value of corresponding registers i.e. genetic diversity is respected. For example, in one generation, tournament selection, two-point crossover, and general replacement may be used and in next generation roulette salaction, one-point crossover, and steady-state replacement be used. This diversity is inplem d neither in [15] nor in [16].

\section{CONCLUSION}

Genetic algorithm is an attractive method that used to observed that by passing through some generation, it may be were produced in some generation ago. Therefore somen thod required to solve this problem. By putting ancestor information of each prod accuchromos in its structure, this difficulty can be curable. From another respective, onetic algorithm jo usually implemented in software and less works have been done to imple nt this algo ithm in hardware. The main and novel contribution of this work is implementin 1 most all of the genetic operators that results in genetic diversity without producino of itera. that work on hardware genetic algorithm, so these operators are implemented and some of them are not implemented in hardware. To eva ura iness measurements, neural network has been used and shown area reauired usin this approach is significantly decreased in compare to previous works. In sy ite a revious vorks, proposed architecture preserve genetic diversity that causes to speed in of architecure. From evaluation results can be drawn a conclusion that by changi oren specific conditions, convergence speed is significantly increased or examp, when some chromosome are produced in alternate generations, changiv o voint cro, over to two-point decreases production of these chromosome and ypove g tic diversity and convergence speed. Evaluation results show advantage of or proposed arch fure over previous works.

\section{Refer}

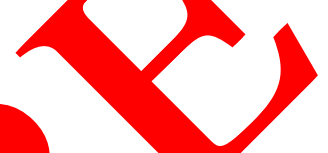

[1. ribo $\Delta$ hmad, Amir Shikh Ahmadi, Intenational Journal of Computer Applications 3. v) (2011) 46-50.

[2] Faribo Amadi, Reza Tati, New hardware engine for genetic algorithm, In Proc $5^{\text {th }}$ International Conference on Genetic and Evolutionary Computing, 2012

[3] Liu J., A general purpose hardware implementation of genetic algorithms, MSc. Thesis, University of North Carolina, 1993.

[4] Scott S. D., Samal A., Seth S., HGA: a hardware-based genetic algorithm, In Proc. ACM/SIGDA 3rd. International Symposium in Field-Programmable Gate Array, pp. 53-59, 1995. 
[5] Turton B. H., Arslan, T., A parallel genetic VLSI architecture for combinatorial real-time applications - disc scheduling, In Proc. IEE/IEEE International Conference on genetic Algorithms in Engineering Systems, pp. 88-93, 1994.

[6] Bland I. M., Megson G. M., Implementing a generic systolic array for genetic algorithms. In Proc. 1st. On-Line Workshop on Soft Computing, pp 268-273, 1996.

[7] Megson G. M., Bland I. M., Synthesis of a systolic array genetic algorithm. In Proc. 12th. International Parallel Processing Symposium, pp. 316-320, 1998.

[8] D. C. Goldberg. Genic algorithm in search, optimization, and machine learning *on Welsey, 1989.

[9] Gaines B. R., Advances in Information Systems Science 2 (1969) 37-172

[10] Nedjah, N., Mourelle, L.M., Lecture Notes in Computer Science 2607 (2003)1

[11] Bade S. L. M., Hutchings B. L., FPGA-Based Stochastic Neurd Implementation, IEEE Workshop on FPGAs for Custom Co r1puting Ca, April 10-13, pp. 189-198, 1994.

[12] Brown B. D., Card H. C., IEEE Transactions on Cora ters 3 (2001) 891-905.

[13] Xilinx, http://www.xilinx.com/, 2004.

[14] Michalewics Z., Genetic algorithms + data str tures = evo ution programs, Springer-Verlag, Berlin, Second Edition, 1994

[15] Scott S. D., Seth S., Samal A., A hard rengine Jurletic algorithms, Technical Report, UNL-CSE-97-001, University thra-Lincoln, July 1997.

[16] N. Nedjah, Pararllel evolutionary compu lons, springer 2006.

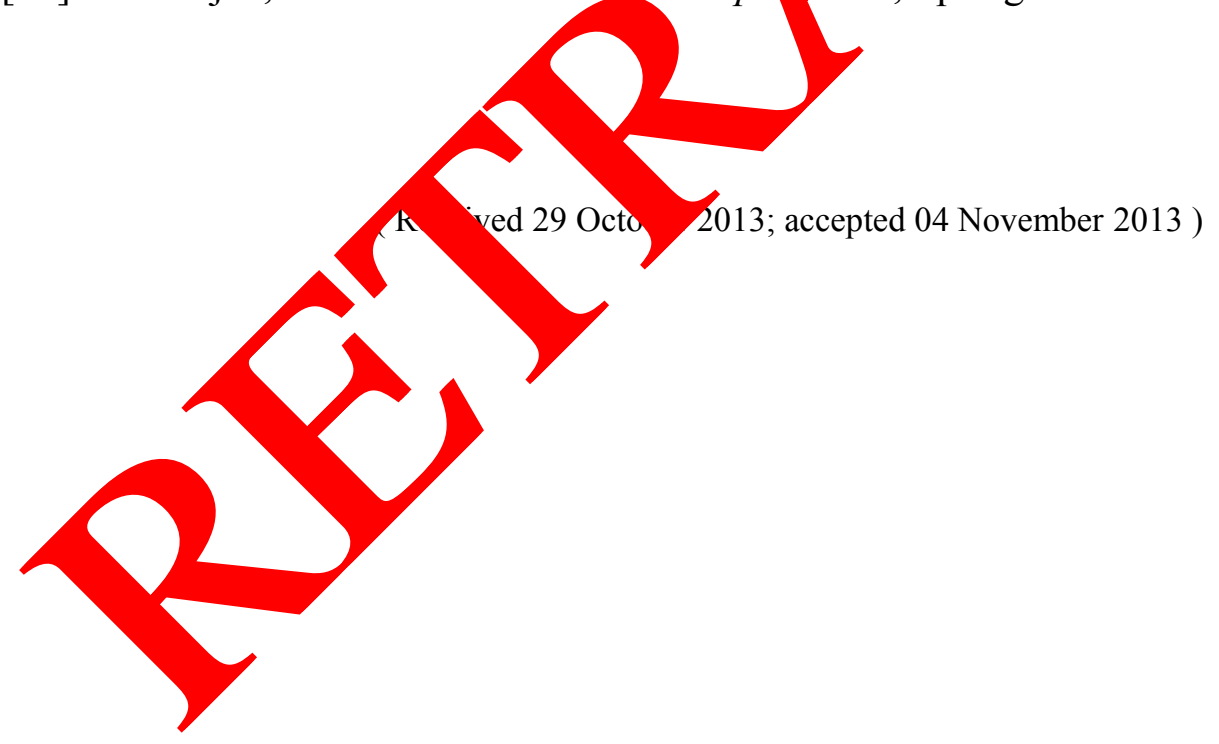

